\title{
EDITORIAL
}

\section{¿POR QUÉ UNA UNIDAD DEL SUELO PÉLVICO?}

\author{
José María Martínez-Sagarra Oceja y Luis Antonio Rodríguez-Toves.
}

Servicio de Urología. Hospital Río Hortega. Valladolid. España.

\begin{abstract}
En muchas ocasiones la Medicina avanza al ritmo de la demanda de las necesidades de la sociedad en la que está inmersa.

En este sentido, el mundo occidental está asistiendo a dos fenómenos importantes. De un lado existe una clara tendencia al envejecimiento de la población que se acentuará aún más en las décadas venideras. Esta situación traerá consigo un cambio profundo en las patologías de la población y por lo tanto en las necesidades de la sociedad, con el consiguiente cambio en la forma de actuar de los profesionales de la salud.
\end{abstract}

Por otra parte, el hecho de vivir en el llamado estado del bienestar induce a que la sociedad en general y el individuo en particular aspiren a unos estándares de vida cada vez mejores: no se trata tan solo de vivir más años sino de la búsqueda de la calidad de vida en todo ese tiempo.

Pues bien, uno de los campos en los que tiene una incidencia clara estos dos hechos antes citados es el de las patologías del suelo pélvico que también son denominadas globalmente como disfunciones del suelo pélvico y que conceptualmente abarcan campos como incontinencia urinaria y fecal, prolapso de órganos pélvicos, anomalías sensitivas y del vaciado del tracto urinario inferior, disfunciones defecatorias, disfunciones sexuales femeninas y síndromes dolorosos crónicos pelvianos.

\section{INCIDENCIA DE LAS DISFUNCIONES DEL SUELO PÉLVICO}

La incidencia de estas disfunciones ha sido objeto de múltiples estudios en el caso de la incontinencia urinaria. Los diversas publicaciones sobre este padecimiento en nuestro país arrojan cifras muy variadas, pero en general se admite que la incontinencia urinaria de esfuerzo afecta predominante- 
mente a mujeres entre los 20 y 50 años de edad y que los síntomas de incontinencia por urgencia son más frecuentes conforme aumenta la edad de las pacientes, especialmente a partir de los 65 años. En cualquier caso, se estima que tan sólo el $25 \%$ de los incontinentes consultan al médico por su problema achacándose a la vergüenza y a la falta de educación sanitaria la falta de dichas consultas. La OMS estimó que en 2003 el número de pacientes con incontinencia urinaria en España era de más de 3 millones doscientas mil personas. El coste que supone esta patología no es fácilmente cuantificable, pero se calcula que el gasto en absorbentes para nuestro sistema nacional de salud viene a ser el $3,2 \%$ del total de la prestación farmacéutica (unos 210 millones de euros en 2001). A esto se debería de sumar el gasto en medicamentos, en consultas médicas, etc.

No existen demasiados estudios sobre la incidencia de prolapsos de órganos pélvicos. Citaremos algunos de especial importancia. En Suecia, Samuelsson y cols realizaron un trabajo sobre 641 mujeres de 20 a 59 años y encontraron una prevalencia de POP del $30,8 \%$ con una incidencia del $2 \%$ de mujeres con prolapso significativo. En Croacia Strinic y cols estudiaron a 1749 mujeres y no encontraron diferencias en la prevalencia del POP entre mujeres del medio urbano o rural. En Corea, Kim y cols estudiaron a 244 mujeres con POP y 314 sin POP y describen que l edad, la paridad y la menopausia son factores de riesgo para el POP. Finalmente, Handa y cols realizaron un estudio en Baltimore (EEUU) en 412 mujeres, concluyendo que el 31,8\% de ellas presentaban POP. Todos estos trabajos nos sugieren que la incidencia del POP trasciende a los países y razas, además de presentar unas cifras respetables de incidencia.

Pero no es menos cierto que en otras patologías como por ejemplo la disfunción sexual femenina, se desconoce con exactitud el número de pacientes que pueden padecerlas. No obstante, se sospecha que dicha incidencia es más alta que el número de pacientes que consultan por ellas.

En su estudio, DeLancey afirma que 1 de cada 10 mujeres tendrá una disfunción del suelo pélvico de tal importancia como para requerir cirugía correctora. Este autor, ante la magnitud del problema actual y futuro afirma que es un problema de salud pública de primer orden y califica a las disfunciones del suelo pélvico como "la epidemia oculta".

Si bien estas patologías no son amenazantes para la vida per se, sí que suponen un gasto económico importante para la sanidad y, en definitiva, para la sociedad que la sustenta en términos de absorbentes para la incontinencia urinaria, consultas y tratamientos debidos a patologías derivadas por estas patologías como depresiones, traumatismos por caídas, infecciones urinarias, etc.

\section{LA PROBLEMÁTICA DE LOS TRATAMIENTOS}

Sin embargo, la aproximación terapéutica de estas patologías ha tenido "mala prensa" desde tiempo inmemorial debido a los malos resultados funcionales que las cirugías ofrecían a estas pacientes. En nuestra opinión esto puede ser debido a varias causas:

- Inadecuada indicación quirúrgica motivada por diagnósticos erróneos.

- Tratamientos mal revisados (estudios retrospectivos sesgados).

- Cirugías "esporádicas" realizadas por profesionales con poca experiencia en el tema debido a que no poseían suficiente casuística.

- Ausencia de una investigación básica o experimental.

Debido a esto, se ha calculado que el $33 \%$ de las intervenciones quirúrgicas por disfunciones del suelo pélvico son reintervenciones por malos resultados de procedimientos quirúrgicos previos. 


\section{LA SOLUCIÓN: LAS UNIDADES DE SUELO PÉLVICO}

Por todas estas circunstancias se han ido formando las unidades del suelo pélvico en algunos centros hospitalarios de nuestro país. Su objetivo principal es el abordaje integral de las patologías/disfunciones del suelo pélvico y abarcan los campos arriba citados.

Para ello, además del personal médico (urólogos, ginecólogos, colo-proctólogos/cirujanos, rehabilitadores, anestesiólogos y radiólogos) debe contar con enfermeras, matronas, fisioterapeutas y psicólogos/sexólogos.

Las actividades de estas unidades abarcan consultas médicas, estudios urodinámicos, procedimientos quirúrgicos, técnicas de vanguardia como la neuromodulación sacra, consultas de cateterismos (enfermería), tratamientos como fisioterapia, procedimientos como PTNS o EMDA, etc. Pero no sólo es importante la labor asistencial, sino que debe complementarse con acciones formativas de otros sanitarios (médicos residentes, estudiantes de enfermería y matronas, entre otros) y de educación sanitaria y prevención de la población general. Por último, y no menos importante, deben llevarse a cabo estudios retrospectivos y ensayos prospectivos dentro de la unidad a fin de mejorar la asistencia sanitaria y aumentar el estándar de calidad de dicha unidad.

La creación de estas unidades tiene la cobertura legal establecida en la Ley 44/2003 de 21 de noviembre denominada Ley de Ordenación de las Profesiones Sanitarias. Dicha Ley en sus artículos 24, 25 y 29 establece el cauce para la obtención del Diploma en las denominadas Áreas de Capacitación Específica que en el caso de nuestra Especialidad abarcaría el campo de la Neuro-uro-ginecología y que supone un marchamo de calidad de cara a la sociedad y para los profesionales que trabajen en dichas unidades.

\section{LAS VENTAJAS DE LAS USP}

El beneficio de la creación de estas unidades es claro y afecta a los pacientes, a la sociedad y a los profesionales sanitarios.

A los pacientes porque la existencia de profesionales bien formados en el campo y con experiencia en el tema redunda en una mejora en la calidad de la asistencia sanitaria y por lo tanto en el calidad de vida de las personas.

A la sociedad, porque con un personal mejor especializado de estas unidades se podría reducir el porcentaje de reintervenciones quirúrgicas. Las actividades de prevención también suponen una disminución de estas disfunciones a la larga y la formación del personal sanitario puede disminuir el gasto en tratamientos como pañales y absorbentes. Como puede deducirse el beneficio para la sociedad es claro en términos económicos y de morbilidad.

Para los profesionales, porque la posesión de un título de Diplomado en el Área de Capacitación Específica de Neuro-uro-ginecología (en el caso de nuestra Especialidad) supone un reconocimiento profesional y una garantía de calidad en la asistencia para la sociedad, además de constituir un mérito en la obtención de los distintos grados en la carrera profesional.

Por todo lo expuesto, consideramos del máximo interés la creación de unidades del suelo pélvico en los centros sanitarios de referencia del país.

\section{BIBLIOGRAFÍA}

1. Bump RC and Norton PA. Epidemiology and natural history of pelvic floor dysfunction. Obstet Gynecon Clin North Am, 1998; 25:723-46.

2. DeLancey JO. The hidden epidemic of pelvic floor dysfunction: achievable goals for improved prevention and treatment. Am J Obstet Gynecol, 2005; 192:1488-95.

3. South M and Amundsen CI. Pelvic organ prolapse: a review of the current literature. Minerva Ginecol, 2007; 59:601-12. 